\title{
Science Autonomy and the ExoMars Mission: Machine Learning to Help Find Life on Mars
}

\author{
V.DA POIAN ${ }^{1,3}$, E. I. LYNESS ${ }^{1,2}$, W. B. BRINCKERHOFF ${ }^{1}$,
} R. DANELL ${ }^{4}, X$. LI $^{1,5}$, M. G. TRAINER ${ }^{1}$

${ }^{1}$ NASA GSFC, Code 699, Greenbelt, MD 20771, USA

victoria.dapoian@nasa.gov

${ }^{2}$ Microtel LLC, Greenbelt, MD, 20770, USA

${ }^{3}$ SURA, CRESST, Washington DC, USA

${ }^{4}$ Danell Consulting Inc, Wintervill, NC, USA

${ }^{5}$ University of Maryland Baltimore County, Baltimore, MD, USA

Planetary missions return exactly one thing: data. The volume of data returned from geochemical instruments on the surface of distant planets is very. Meanwhile, the science instruments that study the geochemistry of planetary bodies generate increasingly large amounts of data. Soon they will generate far more data than can be returned to Earth. We envision smart instruments that analyze science data in-situ such that they can adjust and tune themselves, select the next operations to be run and return to Earth only the most promising results.

We present the first step in this vision: a machine learning (ML) approach for analyzing science data from the Mars Organic Molecule Analyzer (MOMA) instrument, to be delivered to Mars as part of the ExoMars mission in 2023. MOMA, a dual-mode LDMS (Laser Desorption Mass Spectrometer) and GCMS (Gas Chromatographer Mass Spectrometer) mass spectrometer, will search for life, past or present, on the Martian surface and subsurface through analysis of soil samples. We use data collected from the MOMA engineering model to develop mass-spectrometryfocused machine learning techniques.

We first apply unsupervised algorithms in order to cluster input data based on intrinsic patterns and separate the bulk data into clusters. Then, optimal classification algorithms designed for MOMA's scientific goals provide information to the scientists about the likely content of the sample. This will help the scientists in their analysis of the sample and decision-making process about the next operations to conduct.

This is the first step in a longer-term goal in which the spacecraft and instruments themselves make real-time instrument adjustments on operations as we continue to search for life in our solar system and beyond. 